\title{
LE CARCINOME MUCOÉPIDERMOÏDE DE LA PAROTIDE : A PROPOS DE 5 CAS
}

\section{MUCOEPIDERMOID CARCINOMA OF PAROTID GLAND}

\author{
S. KHARRAT, G. BESBES, S. TRABELSI, D. MENIF, A. MEKNI*, \\ H. BEN REHOUMA, S. SAHTOUT, N. BELTAIEF, * M. ZITOUNA*, S. HACHICHA. \\ SERVICE ORL LA RABTA TUNIS \\ *SERVICE D'ANATOMOPATHOLOGIE LA RABTA TUNIS
}

\begin{abstract}
RESUME
Les tumeurs des glandes salivaires représentent moins de $5 \%$ de toutes les tumeurs de la tête et du cou. Parmi eux, le carcinome mucoépidermoïde est la tumeur la plus fréquente. Quarante cinq pour cent des carcinomes mucoépidermoïdes se développent au niveau de la glande parotide. Cette lésion se voit surtout chez l'adulte vers la 5ème décade. Le diagnostic est confirmé seulement à l'examen anatomopathologique.

Son pronostic est fonction des critères histologiques.
\end{abstract}

\section{SUMMARY}

Salivary gland tumors account for less than $5 \%$ of the head and neck neoplasms. Among them, mucoepidermoid carcinoma is the most common malignant salivary gland tumor. About $45 \%$ of mucoepidermoid carcinomas occur in the parotid gland, and appear around the fifth decade of life.

A certain diagnostic can be obtained only after post-operative histological examination. Histological examination indicates the patient prognosis.

\section{INTRODUCTION}

Le carcinome mucoépidermoïde représente 1 à $4 \%$ de l'ensemble des tumeurs parotidiennes. II s'agit de la tumeur maligne la plus fréquente de la glande parotide.

Il peut être observé à tout âge avec une égalité entre les 2 sexes.

II s'agit d'une tumeur à croissance lente avec une symptomatologie non spécifique.

Le traitement est essentiellement chirurgical. Son pronostic dépend des caractères histologiques de la tumeur

\section{MATÉRIEL ET MÉTHODES}

II s'agit d'une étude rétrospective portant sur 5 cas de carcinomes mucoépidermoïdes de la parotide colligés au service ORL et CMF de la Rabta sur une période de 10 ans (19942004).

Tous nos patients ont bénéficié d'un examen clinique cervicofacial et général soigneux ainsi que d'une échographie cervicale. Une tomodensitométrie a été pratiquée dans deux cas. Tous les malades ont été opérés. Une parotidectomie totale a été réalisée dans tous les cas associée à un curage triangulaire systématique. Quatre patients ont eu une radiothérapie complémentaire.

\section{RÉSULTATS}

Notre série comporte 5 patients ayant un âge moyen de 63 ans (45 - 75). Une nette prédominance masculine a été notée avec un sex ratio égal à 4 .
Le délai moyen de consultation était de 4 ans (6mois - 8ans). Tous nos patients ont consulté devant l'apparition d'une tuméfaction de la loge parotidienne.

Une augmentation rapide de la taille de la tumeur a été signalée dans 3 cas avec notion d'algies dans 1 cas et de paralysie faciale dans un autre cas.

A l'examen, on a mis en évidence une tuméfaction de la loge parotidienne dans tous les cas dont la taille variait de 2 à $7 \mathrm{~cm}$ et qui était de consistance ferme dans tous les cas et fixée dans 2 cas.

A la palpation cervicale, on a retrouvé des adénopathies de la zone II-III dans 2 cas. Celles-ci étaient fermes mobiles et indolores avec une taille allant de 1,5 à $2 \mathrm{~cm}$.

Un patient présentait une masse tumorale fixée avec une peau en regard infiltrée et ulcérée associée à une paralysie faciale périphérique grade $V$ de HOUSE.

Une échographie parotidienne a été pratiquée dans tous les cas mettant en évidence une masse hypoéchogène dans 3 cas, solide et hétérogène dans 2 cas. Cet examen a été complété d'une tomodensitométrie chez deux patients montrant une masse polylobée prenant le produit de contraste de façon hétérogène (fig. 1), avec présence de calcification dans un cas. Une exérèse chirurgicale a été réalisée dans tous les cas. L'examen histologique en extemporané avait conclu à la malignité dans tous les cas. Une parotidectomie totale a été réalisée dans tous les cas. Dans un cas, le nerf facial était envahi par la tumeur et un sacrifice du nerf a été pratiqué. Tous les malades ont eu un curage triangulaire emportant les 
ganglions de la zone II-III.

L'examen anatomopathologique définitif a conclu à un carcinome mucoépidermoïde (figure $\mathrm{n}^{\circ} 2$ ) de grade intermédiaire dans 4 cas et de haut grade de malignité dans un cas.

Une radiothérapie post opératoire a été réalisée chez 4 malades à la dose de 40 à 60 Gray.

Le recul moyen de nos patients est de 2 ans. Un cas de réciudive ganglionnaire a été noté, à un an post radiothérapie. Une radiothérapie de rattrapage lui a été indiquée.

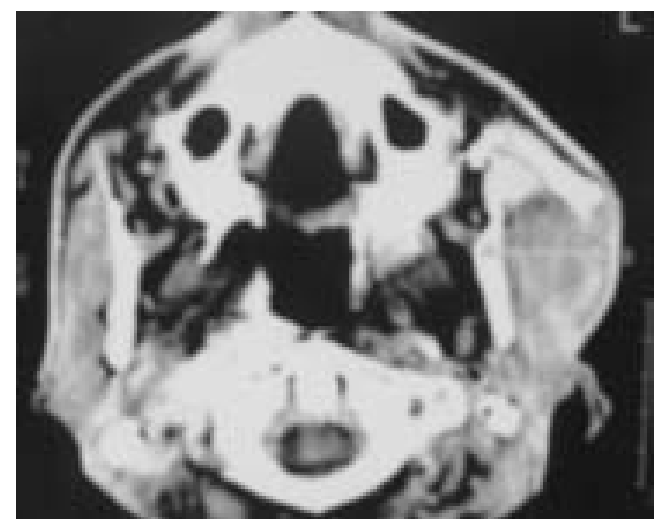

Fig. 1 :TDM en coupe axiale : tumeur aux dépens de la glande parotide gauche se rehaussant de façon hétérogène après injection de PDC

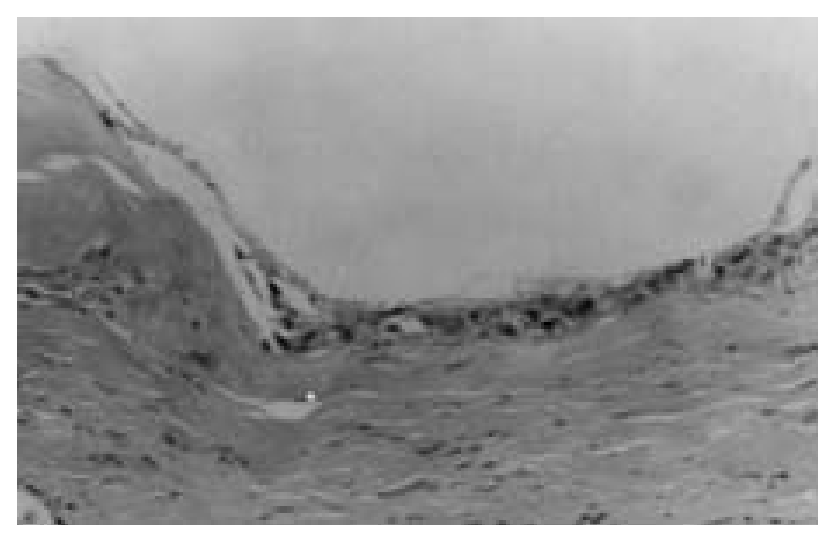

Fig. 2 : Cavité kystique revêtue par des cellules mucosécrétantes et des cellules épidermoïdes

\section{DISCUSSION}

Décrit pour la première fois par Stanly en 1988, le carcinome mucoépidermoïde (CME) représente moins de $10 \%$ des tumeurs des glandes salivaires, et prés de $30 \%$ des tumeurs malignes des glandes salivaire $(1,2,3)$.

La localisation parotidienne est la plus fréquente 64 à $80 \%$ $(1,4,5,6)$.

L'âge de survenue se situe entre la $3^{\text {ème }}$ et $5^{\text {ème }}$ décade $(5,6$, 7). Toutefois, certains auteurs ont rapporté des cas chez l'enfant. Claros a rapporté un cas de CME de la parotide chez un enfant de 4 ans (8) alors que Horst a rapporté un cas de CME de bas grade chez un garçon de 8 ans (7).

Cette tumeur se voit aussi bien chez l'homme que chez la femme sans aucune prédominance de sexe.

Le lipiodol et les irradiations ont été incriminés dans la genèse de cette tumeur. Toutefois, il n'existe pas de corrélation avec le tabagisme $(1,5,6)$.

Sur le plan histologique, le carcinome mucoépidermoïde se présente sous forme de lobules à double différenciation, composé de cellules glandulaires renfermant du mucus, de cellules épidermoïdes et des cellules intermédiaires capables de se différencier en cellules épidermoïdes ou en cellules glandulaires $(3,5)$.

On distingue 3 types histologiques :

- Le carcinome mucoépidermoïde bien différencié de bas grade de malignité.

- Le carcinome mucoépidermoïde peu différencié de haut grade de malignité.

- Le carcinome mucoépidermoïde de différenciation modérée: de malignité intermédiaire.

Des études récentes en immunohistochimie ont montré l'expression d'un gène codant pour des molécules d'adhésion inter-cellulaires appelé MEL-CAM (CD146) présent sur le chromosome 11 dans $92,7 \%$ des cas (5).

Son expression est plus importante en fonction du grade de malignité. Plus le grade est grand, plus son expression est forte.

Sur le plan clinique, le carcinome mucoépidermoïde réalise souvent une tumeur parotidienne solitaire et mobile rappelant l'adénome pléomorphe, de croissance lente et indolore de petite taille $(2-3 \mathrm{~cm})(6,7)$, avec parfois des poussées évolutives, et augmentation rapide du volume et fixation aux tissus avoisinants, sans douleur ni paralysie faciale.

Les examens morphologiques, dominés par l'échographie, montrent une lésion kystique sans signes spécifiques de malignités. La tomodensitométrie (TDM) et l'IRM sont indiquées dans le cadre d'un bilan d'extension, en cas de tumeur du lobe profond ou en cas de tumeur non palpable cliniquement. Le diagnostic de malignité peut être suspecté par la cytoponction et le PET- scan, mais c'est l'exploration chirurgicale avec étude histologique de la pièce, qui constitue la clef du diagnostic $(7,8)$.

Le traitement du carcinome mucoépidermoïde de la parotide est basé sur la chirurgie et la radiothérapie $(2,3)$.

Le traitement peut être conservateur se limitant à une parotidectomie exofaciale ou sub-totale si la tumeur n'atteint que le lobe superficiel, en cas de tumeur de bas grade ou de grade intermédiaire avec absence d'adénopathies cliniquement et échographiquement $(8,9)$.

Le traitement chirurgical doit être radical associant une parotidectomie totale et un évidemment ganglionnaire cervical en cas de tumeur de haut grade de malignité, en cas d'envahissement nerveux ou vasculaire et en cas d'adénopathies.

La radiothérapie est formelle dans les formes de haut grade de malignité, et discutée dans les formes de bas grade et de malignité intermédiaire.

Le pronostic dépend des caractères histologiques de la tumeur. Il est meilleur pour les carcinomes de bas grade $(6,7)$. 
Les facteurs histologiques de gravité sont : le degré de différenciation cellulaire, la richesse en cellules muco-sécrétantes, l'importance des mitoses, la présence de nécrose, l'invasion nerveuse et vasculaire et l'infiltration des structures avoisinantes $(8,9)$.

\section{CONCLUSION}

Le carcinome mucoépidermoïde représente la tumeur maligne la plus fréquente de la glande parotide. Son diagnostic est histologique. Cette tumeur est caractérisée par différents grades de malignité qui conditionne son pronostic.
Le traitement est chirurgical associé à une radiothérapie essentiellement pour les formes de haut grade de malignité.

\section{REFERENCES}

1-ARMTONG J., HARISSON L.SPINO, R.. Tumeurs malignes des glandes salivaires principales : intérêt de l'association radio-chirurgicale. Arch. Otolaryngol 1990, $9: 149-52$.

2- BELLOC J.B., LACCOURREYE O., CHABARDES E.. Les tumeurs mucoépidermoïdes de la parotide. Attitude diagnostique et thérapeutique. Ann. Otolaryngol 1991, 108: 119- 25

3- ESSADI M., RAJI A., JAUCH A., KADIRI F. et AL. Les tumeurs de la parotide (à propos de 120 cas). Rev laryngol otol rhinol 1998, $119: 177-82$.

4- TARY M., ESSADI M., RAJI A.,AIT BEN HANOU C.. Les tumeurs de la parotide, aspect diagnostics, et thérapeutiques : à propos de 145 cas. Magreb médical $2000,20: 6-9$

5- RAMOA F., IE-MING SHIH, CRUZ PEREZ D. E.

Mel-CAM (CD146) expression in parotid mucoepidermoid carcinoma. Oral Oncolgy, 2003 vol $39: 277-281$.

6- KOFI D., BOAHENE O., KERRY D, LEWIS J... The Mayo clinic experience : Mucoepidermoid carcinoma of the parotid gland. Arch Otolaryngol Head Neck
Surg. 2004; $130: 134$.

7- KORKEMUELLER H., BRUEGGEMANN N., SWENNEM G., ANDRE ECKARDT. Mucoepidermoid carcinoma of the salivary glands clinical review of 42 cases. Oral Oncology 2005; 41, 1: 3-10.

8-. CLAROS P, DOMINTE G., CASTILLO JR M., CARDES AN., CLAROS A.. Parotid gland mucoepidermoid carcinoma in a 4-year-old child International Journal of Pediatric Otorhinolaryngology 2002; 63: 67-72

9- YOSHIHARA T. , SUZUKI S., NAGAO K. et al. Mucoepidermoid carcinoma arising in the accessory parotid gland. International Journal of Pediatric Otorhinolaryngology 1999, $48: 47-52$ 